\title{
C1GALT1 expression predicts a favorable prognosis and suppresses malignant phenotypes via TrkA signaling in neuroblastoma
}

\author{
Neng-Yu Lin ${ }^{1}$, Syue-Ting Chen ${ }^{1,2,3}$, Hsiu-Ling Chang ${ }^{1}$, Meng-Yao Lu ${ }^{4}$, Yung-Li Yang ${ }^{4,5}$, Shu-Wei Chou ${ }^{4}$, Dong-Tsamn Lin ${ }^{4,5}$, Kai-Hsin Lin ${ }^{4}$, \\ Shiann-Tarng Jou ${ }^{4}$, Wen-Ming Hsu ${ }^{6}$, Min-Chuan Huang ${ }^{1}{ }^{1}$ and Hsiu-Hao Chang (D ${ }^{4 凶}$
}

(C) The Author(s) 2022

Neuroblastoma (NB) is a childhood tumor derived from the sympathoadrenal lineage of the neural crest progenitor cells. Core $1 \beta 1,3$-galactosyltransferase (C1GALT1) controls the crucial step of GalNAc-type O-glycosylation, and its altered expression affects cancer behaviors. However, the role of C1GALT1 in NB tumors remains unclear. Our data showed that C1GALT1 expression was significantly associated with differentiated tumor histology, correlated with TrkA expression, and predicted good prognosis independently in NB. Downregulation of C1GALT1 promotes malignant behaviors of NB cells in vitro and in vivo. Mechanistic investigation showed that knockdown of C1GALT1 in NB cells increased TrkA pulled down through Vicia villosa agglutinin beads, indicating the modulation of O-glycans on TrkA by C1GALT1, and silencing C1GALT1 suppressed the TrkA expression on the NB cell surface. Overexpression of C1GALT1 increased the protein levels of TrkA and promoted the differentiation of NB cells, whereas knockdown of TrkA inhibited C1GALT1-induced neuronal differentiation. Moreover, the inhibitory effects of migration and invasion in C1GALT1-overexpressing NB cells were blocked by TrkA downregulation. C1GALT1 knockdown enhanced AKT phosphorylation but attenuated ERK phosphorylation, and these properties were consistent in C1GALT1-overexpressing NB cells with TrkA knockdown. Taken together, our data provided the first evidence for the existence of GalNAc-type O-glycans on TrkA and altered O-glycan structures by C1GALT1 can regulate TrkA signaling in NB cells. This study sheds light on the novel prognostic role of C1GALT1 in NB and provides new information of C1GALT1 and TrkA on the pathogenesis of NB.

Oncogenesis (2022)11:8; https://doi.org/10.1038/s41389-022-00383-w

\section{INTRODUCTION}

Neuroblastoma (NB) is a childhood tumor derived from sympathoadrenal lineage of the neural crest progenitor cells. It develops in the neurons of sympathetic nervous system, and in the medulla of adrenal gland. It occurs with an incidence of 8.0 per million per year [1], and $96 \%$ of cases occur before the age of 10 years [2]. NB represents the most common extracranial solid tumor in children as well as the most frequently diagnosed malignancy during infancy [3]. The clinical presentation of NB can be categorized into three distinct patterns: (i) life-threatening progression and metastasis; (ii) maturation to ganglioneuroblastoma (GNB) or ganglioneuroma (GN); and (iii) spontaneous regression [4]. So NB is quite a heterogeneous tumor and presents a broad clinical and biologic spectrum ranging from highly undifferentiated tumors with very poor outcomes to the most differentiated benign GN or NB with spontaneous regression and hence favorable prognosis [3]. Although NB represents $5-8 \%$ of all malignant childhood diseases, it is disproportionately responsible for up to $10-15 \%$ of cancer deaths in children [5].
NB patients can be classified into different risk groups for clinical treatment based on relevant biologic prognostic factors $[6,7]$. Among these factors, MYCN proto-oncogene amplification, which is detected in about $20-30 \%$ of NB, is considered as the most reliable genomic hallmark of aggressive tumor behavior and treatment failure $[3,6,8,9]$. MYCN expression is well known to be suppressed in differentiating NB cells by TrkA activation with its ligand nerve growth factor (NGF) [10]. TrkA is one of the members of the Trk family which are receptor tyrosine kinase (RTKs) [10]. The Trk family receptors are initially synthesized as precursor proteins; the post-translational glycosylation of the extracellular domains of these precursors is required to localize TrkA to the cell surface, where it can trigger the Ras/MAP/ERK kinase cascade for neurotrophin-promoted differentiation of neurons [11-14].

Glycosylation is one of the most common posttranslational modifications of proteins in mammalian cells and alterations in glycosylation regulate the development and progression of cancer [15]. Altered glycan structures on cell surfaces are prominent features of cancer cells and play important roles to

\footnotetext{
${ }^{1}$ Graduate Institute of Anatomy and Cell Biology, National Taiwan University College of Medicine, Taipei, Taiwan. ${ }^{2}$ Department of Anatomy, College of Medicine, Chang Gung University, Taoyuan, Taiwan. ${ }^{3}$ Department of Neurosurgery, Chang Gung Memorial Hospital, Taoyuan, Taiwan. ${ }^{4}$ Department of Pediatrics, National Taiwan University Hospital,

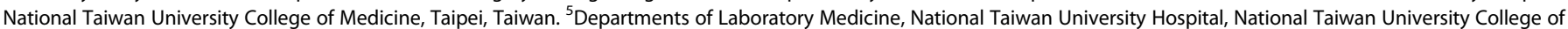
Medicine, Taipei, Taiwan. ${ }^{6}$ Department of Surgery, National Taiwan University Hospital, National Taiwan University College of Medicine, Taipei, Taiwan.

email: changhh2001@ntu.edu.tw
}

Received: 23 October 2021 Revised: 29 January 2022 Accepted: 2 February 2022

Published online: 15 February 2022 
affect cell behaviors. GalNAc-type O-glycosylation is the most common type of O-glycosylation, and it modulates diverse functions of membrane-bound and secreted proteins [16]. During GalNAc-type O-glycosylation, the GALNT family enzymes transfer N-acetylgalactosamine (GalNAc) from UDP-GalNAc to serine (Ser) or threonine (Thr) residues to form the GalNAC-OSer/Thr structure, also known as Tn antigen [17]. Subsequently, core $1 \beta 1,3$-galactosyltransferase (C1GALT1), an exclusive T-synthase in mammalian cells, catalyzes the transfer of galactose (Gal) from UDP-Gal to the Tn antigen, forming GalGalNAc-O-Ser/Thr structure, known as $T$ antigen or core 1 structure. The core 1 structure is a basis for further complex O-glycan formation, such as core 2 structure [18]. CIGALT1 plays critical roles in many biological functions, and its deletion results in developmental defects, spontaneous colitis and thrombocytopenia in mice [18-21]. Moreover, aberrant expression of truncated O-glycans is a characteristic mark observed on the surface of tumor cells, and is associated with an adverse outcome and poor prognosis in patients with several malignant diseases [22]. Previous studies showed that C1GALT1 is overexpressed in hepatocellular carcinoma, colorectal cancer, breast cancer, head, and neck squamous cell carcinoma, and gastric cancer [23-28]; and increased C1GALT1 expression correlates with higher histological grade, or advanced tumor stage and poor survivals in the above cancers. At the molecular level, manipulating the expression of C1GALT1 has been demonstrated to regulate O-glycosylation of MET in hepatocellular carcinoma, FGFR2 in colorectal cancer, Mucin 1 in breast cancer, EGFR in head and neck squamous cell carcinoma cells, and EPHA2 in gastric cancer, respectively, and affects cancer malignant behaviors [23, 24, 26-28]. However, unlike the above adult tumors, NB is the most common extracranial solid tumor in children, and rare in adults [29]. The pathophysiologic mechanism of C1GALT1 in NB tumors remains largely unclear.

The present study is the first report to show that high C1GALT1 expression correlates with NB tumor differentiation status and predicts better survival outcomes of patients with NB. The chisquare analysis also indicated positive correlations between the expression of TrkA and C1GALT1 in NB tumors. In addition, downregulation of C1GALT1 promotes malignant behaviors of NB cells in vitro and in vivo by altering TrkA glycosylation and its downstream signaling pathways.

\section{MATERIALS AND METHODS}

Patients and treatment

From December 1990 to December 2020, 134 histologically proved NB patients with complete follow-up were included in this study. All diagnoses of tumors were confirmed by histologic assessment of a specimen obtained from the primary or metastatic tumor at surgery. Based on the criteria of the International NB Pathology Classification [30], the differentiating status of the tumor histology was categorized into undifferentiated NB (UNB, Schwannian stroma poor), differentiating NB (DNB, Schwannian stroma poor, including poorly differentiated subtype), and ganglioneuroblastoma, intermixed (GNB, Schwannian stroma-rich) according to the percentage and degree of differentiation of the NB cells. The nodular type GNB was classified into either undifferentiated or differentiating NB according to the morphology of their NB nodules, since the prognosis of this type of tumors depends mainly on their NB nodules [31]. The distinction of tumor staging was based on the International NB Staging System (INSS) [32]. MYCN status of the tumor tissue was evaluated by chromogenic in situ hybridization analysis of formalin-fixed paraffinembedded tissues or fresh tumor single cells [33]. The treatment of these patients has been described in previous studies [34-36]. The clinical parameters, including age at diagnosis, sex, primary tumor sites, clinical stages, differentiating status of tumor histology, and MYCN amplification status were collected for prognostic and survival analysis. Written consent was obtained from the patients. This study was approved by the Institutional Review Board of the National Taiwan University Hospital (IRB No: 201812005RIND).

\section{Immunohistochemistry}

One hundred eighty-four tumor specimens collected before chemotherapy were fixed in formalin and embedded in paraffin. The expression of C1GALT1 was evaluated by using an avidin-biotin complex immunoperoxidase staining technique as described previously [37]. A monoclonal anti-C1GALT1 antibody (Santa Cruz) was used and signals were detected with Super Sensitive Link-Label immunohisto-chemistry Detection System (BioGenex). The specific staining was visualized with 3,3-diaminobenzidine liquid substrate system (Sigma) and counterstained with hematoxylin (Sigma).

\section{Cell lines and cell culture}

Human NB cells, SK-N-BE, SKN-SH, and GI-LI-N, were kindly provided by Dr. Yung-Feng Liao, Institute of Cellular and Organismic Biology, Academia Sinica, Taipei, Taiwan. These cell lines were cultured with Dulbecco's modified Eagle's medium (DMEM; Invitrogen) containing 10\% FBS (Invitrogen), $100 \mathrm{IU} / \mathrm{mL}$ penicillin, and $100 \mu \mathrm{g} / \mathrm{mL}$ streptomycin (Invitrogen) in a humidified tissue culture incubator at $37^{\circ} \mathrm{C}$ and $5 \% \mathrm{CO}_{2}$ atmosphere. All these cell lines were authenticated by short tandem repeat profiling and negativity of mycoplasma contamination was routinely checked through PCR every week. For stable knockdown and overexpression of C1GALT1 in NB cells, sh-C1GALT1/pLKO.1-puro and sh-control/pLKO.1-puro (RNAi Core, Academia Sinica) were used in the lentivirus-based infection system and stable pooled clones were selected and maintained with $1 \mu \mathrm{g} /$ $\mathrm{mL}$ puromycin (Sigma). The following target sequences were used: shC1GALT1\#1 (5' CCCAGCCTAATGTTCTTCATA $\left.3^{\prime}\right) ;$ shC1GALT1\#2 (5' $^{\prime}$ GCTCTGATCTTGCAGTTTCTT 3').

\section{Quantitative real time-PCR}

Total RNA was purified using NucleoZOL reagent (MACHEREY-NAGEL, Germany) and $1 \mu \mathrm{g}$ RNAs were prepared for CDNA synthesis by MMLV Reverse Transcription kit (Protech Technology Enterprise, Taiwan). Gene expression was quantified by SYBR Green QPCR. The following primer pairs were used: human C1GALT1: 5'-GAAAGTTTGCCTGGGTCCTCT TGG GAGAAA AGGTTGACACC- $3^{\prime}$ and $5^{\prime}$-CTGGGATGCGGAGAGCGCTTGACGTGTTGGCC TाT-3; human TrKA: 5'-AGAACACGCGTATTTACCTGC CCTGCTGGCTTGGCT GATACT-3' and 5'-ACCATGAGTCTAACATCTCCCAC ACGGAGACCACTCTTC ACGA-3'; human $\beta$-actin, $5^{\prime}$-CGTGCGTGACATTAAGGAGA-3' and $5^{\prime}$-GAAGG AAGGCTGGAAGAGTG-3'; $\beta$-actin was used to normalize for the amounts of loaded CDNA.

\section{Western blot analysis and lectin pull-down assay}

Cell lysates were extracted in lysis buffer (Thermo Fisher Scientific). Proteins were separated on a $10 \%$ SDS-PAGE and transferred onto PVDF membranes. The membranes were blocked in TBST containing $5 \%$ bovine serum albumin (Bio-Rad) and then incubated with monoclonal antibodies for C1GALT1 (Santa Cruz Biotechnology), GAPDH (Santa Cruz Biotechnology), TrkA (LSBio), AKT, and pAKT (Ser536) (Cell Signaling Technology) and polyclonal antibodies for ERK1/2 (phospho Thr202/Tyr204, phosphor Thr185/Tyr187) antibody and ERK1/2 (GeneTex) and biotinylated Vicia villosa lectins (VVA or VVL) (Vector Laboratories). After incubation with horseradish peroxidase (HRP)-conjugated secondary antibodies and HRP-conjugated streptavidin, protein bands were detected with ECL reagents (GE Healthcare Life Sciences). For lectin pulldown assay, $500 \mu \mathrm{g}$ of total proteins from cell lysates with or without $0.4 \mathrm{U} /$ $\mathrm{mL}$ neuraminidase (Sigma-Aldrich) treatment were incubated with VVA, Griffonia simplicifolia lectin I (GSL-I), and Peanut agglutinin (PNA) lectinconjugated beads (Vector Laboratories). The beads were washed and boiled to pull down proteins. The samples can be separated by SDS-Polyacrylamide Gel Electrophoresis for western blot analysis.

\section{Immunofluorescence microscopy}

Cells were cultured in chamber slides (SPL Life Sciences), fixed with 4\% PFA, permeabilized with $0.25 \%$ Triton X100, blocked in PBS containing $2 \%$ bovine serum albumin (Bio-Rad) and applied with primary antibodies to TrkA (LSBio), Lamp1 (GeneTex). Alexa Fluor antibodies (Life Technologies) were used as secondary antibodies. Isotype control antibodies were used as controls. Cell nuclei were visualized with DAPI (Santa Cruz Biotechnology). Images were captured using a Nexcope NE950 fluorescent microscope (Ningbo Yongxin Optics Co., United States). 

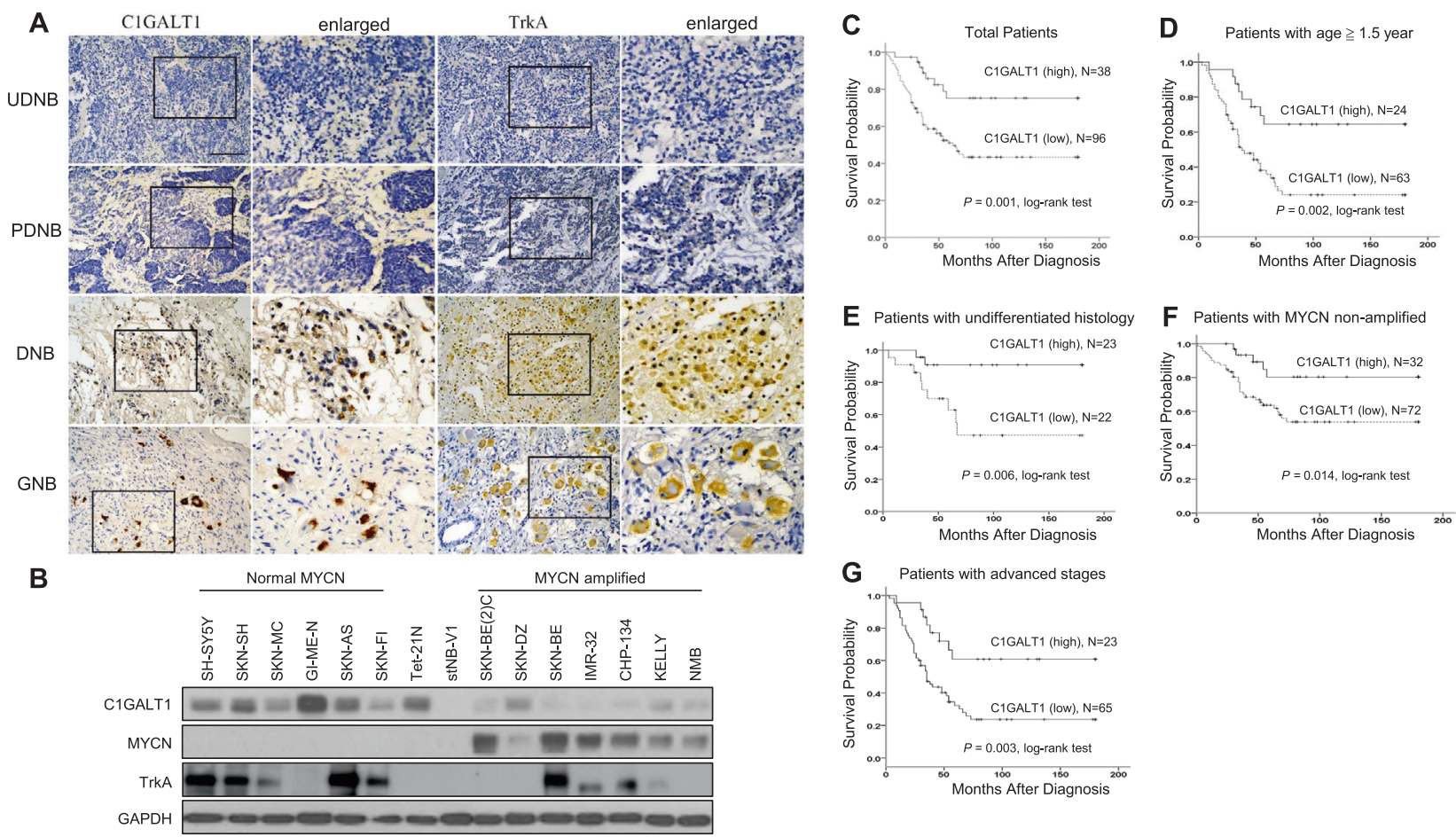

Fig. 1 C1GALT1 expression predicts better prognosis for patients with NB. A Representative images of IHC staining for C1GALT1 protein in NB tumors with indicated histological types. Brown colors represent positively stained cells. Tumors with more mature differentiation (GNB or DNB) exhibit higher expression levels of C1GALT1 protein in general. Inversely, UNB and NB tumors exhibit lower levels of C1GALT1 expression. Scale bars, $100 \mu \mathrm{m}$. High magnified images of the area are marked by the rectangle. B Immunoblotting analysis of C1GALT1, MYCN, and TrkA expression in various NB cell lines with MYCN amplification or non- amplification, using GAPDH as internal control. C Kaplan-Meier overall survival analysis according to the expression of C1GALT1 in 134 patients with NB ( $p=0.001$, log-rank test). D-G Kaplan-Meier survival analysis according to the expression of C1GALT1 in patients with age $\geqq 1.5$ years ( $p=0.002$, log-rank test), undifferentiated histology ( $p=0.006$, log-rank test), MYCN non-amplified $(p=0.014$, log-rank test), advanced-stage disease $(p=0.003$, log-rank test), respectively.

\section{Colony formation assay}

Each group of transfected cells were seeded in six-well plates (500 cells per well) and cultured as indicated. At day 14, cells were washed with PBS, fixed with $4 \%$ formaldehyde, and stained with $0.1 \%$ crystal violet.

\section{Transwell migration and Matrigel invasion assays}

Cell migration and invasion assays were evaluated using transwell (Corning, NY, USA) or Matrigel-coated (BD Biosciences, CA, USA) transwell chamber, respectively. Each transwell chamber contained a membrane filter with pore size $8 \mu \mathrm{m}$. Each group of transfected NB cells $\left(1 \times 10^{5}\right)$ were seeded into the transwell or Matrigel-coated transwell chamber contained with $0.25 \mathrm{~mL}$ serum-free DMEM. After incubating for $48 \mathrm{~h}$, the cells were fixed and stained with $0.5 \%(\mathrm{w} / \mathrm{v})$ crystal violet (Sigma) containing $20 \%$ $(\mathrm{v} / \mathrm{v})$ methanol. The migrated and invaded cells from three random fields were counted under a microscope.

\section{Animals}

All animal experiments were carried out in accordance with a study protocol and guidelines approved by the National Taiwan University College of Medicine and College of Public Health Institutional Animal Care and Use Committee (IACUC). Female NOD-SCID mice aged 6 weeks were purchased from the National Laboratory Animal Center, Taipei, Taiwan. For experimental metastasis analysis, $5 \times 10^{6} \mathrm{SK}-\mathrm{N}-\mathrm{BE}$ and GI-LI-N cells (mock and sh(1GALT1) were intracardially injected into the ventricles. Animals were sacrificed at day $\mathbf{4 0}$ for evaluation of lung metastasis.

\section{Statistical analysis}

The statistical analysis was carried out with SPSS 17.0 for Windows software. Associations between pairs of categorical variables were assessed with Pearson's chi-square test. Survival probabilities in various subgroups were estimated using the Kaplan-Meier method, and analyzed by log-rank tests. The influence of each variable on survival was assessed by the univariate and multivariate Cox proportional hazard model. The significance of the variations between the data resulting from different experiments was analyzed by Student's $t$-test. All statistical tests were twosided, and those with a $p$ value $<0.05$ were considered to be statistically significant.

\section{RESULTS}

High expression of C1GALT1 protein correlates with the differentiation status of NB tumors and predicts better survival outcomes

We examined the C1GALT1 protein expression in 134 NB tumors by immunohistochemical (IHC) staining. Positive C1GALT1 staining was seen in ganglion cells, and localized in the cytoplasm in NB. No schwannian stromal cells showed positive staining of C1GALT1. The immunoreactivity of C1GALT1 was classified into four categories: "-" (no expression, no stained cells or only isolated single stained cells seen), " $1+$ " (weak expression, around $10-35 \%$ of cells stained), " $2+$ " (moderate expression, around $35-70 \%$ of cells stained) and " $3+$ " (strong expression, more than $70 \%$ of cells stained). We found that tumors with more mature differentiation histology (GNB or differentiating NB (DNB)) exhibit higher expression levels of C1GALT1 protein (Fig. 1A). Similar correlations were observed in the Western blot analysis of human NB cell lines, showing the protein levels of C1GALT1 in MYCN-non-amplified NB cell lines are mostly higher than that in MYCN-amplified NB cell lines (Fig. 1B). In addition, high expression (immunoreactivity $2+$ to $3+$ ) of C1GALT1 protein could be detected in 38 of the $134 \mathrm{NB}$ tumors (28.4\%). The majority of NB tumors with high C1GALT1 protein expression were of differentiated histology, differentiated NB cells or ganglion cells. The intensity and percentage of positive C1GALT1 immunostaining correlated strongly with the 
Table 1. C1GALT1 expression and clinicopathologic and biologic characteristics of NB.

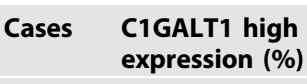

Sex

Male

Female

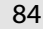

18 (21.4)

$50 \quad 20(40.0)$

Primary tumor site

Adrenal

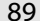

$24(27.0)$

45

14 (31.1)

Age at diagnosis

$\leq 1.5$ year

$>1.5$ year

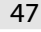

14 (29.8)

87

24 (27.6)

Clinical stage

$\begin{array}{lll}1,2,4 S & 46 & 15(32.6) \\ 3,4 & 88 & 23(26.1)\end{array}$

Tumor histology

Undifferentiated $\mathrm{NB}^{\mathrm{a}}$

Differentiated $\mathrm{NB}^{\mathrm{b}}$

89

15 (16.9)

$<0.001^{*}$

MYCN

$\begin{array}{lrr}\text { Amplified } & 30 & 6(20.0) \\ \text { Non-amplified } & 104 & 32(30.8)\end{array}$

${ }^{*} p<0.05$.

ancluding undifferentiated NB and poorly differentiated NB.

Including differentiating NB and ganglioneuroblastoma.

differentiation of histology. Kaplan-Meier analysis showed that patients with C1GALT1 high expression had a better overall survival rate than those with C1GALT1 low expression (immunoreactivity "-" or "1+") $(p<0.001$, log-rank test) (Fig. 1C).

\section{High expression of C1GALT1 is an independent favorable prognostic marker of NB}

The relationship between C1GALT1 expression and clinicopathologic and biologic variables of NB tumors was summarized in Table 1. Thirty of 134 (22.4\%) NB tumors were recognized as MYCN amplification. Tumors were assigned into C1GALT1 low expression ("-" or " $1+$ " in immunoreactivity) and high expression (" $2+$ " or "3 $+"$ in immunoreactivity) in the statistical analysis. C1GALT1 high expression was significantly associated with differentiated tumor histology $(p<0.001$, Table 1). Furthermore, univariate analysis showed that, in addition to C1GALT1 low expression, patient age at diagnosis $>1.5$ years, advanced clinical stage (INSS stage 3 and 4), MYCN amplification, and undifferentiated histology strongly correlated with poor survival (Table 2). Multivariate analysis revealed that the clinical stage, MYCN amplification, undifferentiated histology, and C1GALT1 low expression remained independent prognostic factors for poor survival (Table 2). To further evaluate the significance of C1GALT1 expression in prognostic discrimination, the impact of C1GALT1 expression on survival probability was analyzed against age of patients, differentiation status of tumor, MYCN amplification status, and clinical stages. Results revealed that C1GALT1 high expression in NB tumors constantly predicted better survival outcomes for patients with age $>1.5$ years (Fig. 1D, $p=0.002$, log-rank test), undifferentiated tumor histology (Fig. 1E, $p=0.006$, log-rank test), MYCN nonamplified (Fig. $1 \mathrm{~F}, p=0.014$, log-rank test), and advanced stages (Fig. 1G, $p=0.003$, log-rank test). In addition, we used SEQC-498 and Asgharzadeh-249 datasets from R2: "Genomics Analysis and Visualization Platform" as independent cohorts to validate our findings of C1GALT1 expression in NB tumors. The analytical results showed that C1GALT1 high expression in NB predicts better survival outcomes compared with C1GALT1 low expression ( $p=$ $1.5 \mathrm{e}-03$ and $p=0.011$, respectively). Furthermore, C1GALT1 high expression in NB tumors correlated with good clinical prognostic factors, such as non-high risk $(p=0.033)$ and MYCN non-amplified $(p=1.45 \mathrm{e}-10)$ status (Supplementary Fig. 1$)$. These are compatible with the findings of C1GALT1 expression in our cohort. All these observations suggest that C1GALT1 expression is an independent prognostic factor for better survival outcomes in NB patients, and could provide complimentary prognostic information in addition to age of patients, differentiation status of tumor, MYCN amplification status, and clinical stages.

\section{C1GALT1 expression correlates with TrkA expression in NB tumors}

Since MYCN expression is well known to be suppressed in differentiating NB cells by TrkA activation with its ligand, NGF, and high TrkA expression in primary NB was associated with favorable clinical features and inversely associated with MYCN amplification $[10,38]$. Furthermore, NGF/TrkA signaling was noted to affect NB differentiation or regression depending on the particular microenvironment [3]. These observations of TrkA activation or expression on NB are compatible with our results for the effects of C1GALT1 expression on NB. We thus investigated if C1GALT1 expression correlates with and affects TrkA expression in NB tumors. We examined the TrkA protein expression in 46 NB tumors by IHC staining and results revealed that TrkA expression significantly $(p=0.001)$ and positively correlated with C1GALT1 expression in NB (Supplementary Table 1).

\section{Downregulation of C1GALT1 promotes malignant behaviors of NB cells in vitro and in vivo}

C1GALT1 was differentially expressed in various kinds of NB cell lines, most notably in MYCN-non-amplified cells (Fig. 1B). To further determine the function of C1GALT1 in NB malignant behaviors, cell proliferation, colony formation, migration, and invasion were analyzed. First, real-time RT-PCR and Western blot analysis confirmed that the mRNA and protein levels of C1GALT1 were significantly downregulated in stable NB cell lines, GI-ME-N, SK-N-BE, and SK-N-AS, on day 20 post transfection (Fig. 2A). The representative bright-field microscopy images and the analysis indicated that C1GALT1 knockdown reduced neurite outgrowth in NB cells (Fig. 2B, C). Supportively, the real-time RT-PCR analysis also showed that C1GALT1 knockdown inhibited mRNA expression of differentiation markers in NB cells (Supplementary Fig. 2). In addition, the colony formation analysis showed that knockdown of C1GALT1 significantly increased colony formation compared with the mock transfectants (Fig. 2D, E). In transwell migration and Matrigel invasion assays, C1GALT1 knockdown increased NB cell migration and invasion (Fig. $2 \mathrm{~F}-\mathrm{I}$ ). These studies suggested that inhibition of C1GALT1 enhances malignant phenotypes in NB cells. We next examined whether this effect also occurs in vivo. GI-ME-N and SK-N-BE cells stably transfected with shC1GALT1 or control lentivirus were intracardially injected into 6-week-old NOD-SCID mice. Animals were sacrificed at day 40 for evaluation of lung metastasis. The results showed that silencing C1GALT1 increased metastatic nodules in the lungs of NOD-SCID mice received GI-ME$\mathrm{N}$ and SK-N-BE transfectants (Fig. 2J).

C1GALT1-mediated 0-glycans regulate cell surface expression and signaling of TrkA in NB cells

We have identified that multiple RTKs, such as EGFR, FGFR2, EPHA2, and MET, are key targets for C1GALT1 and their appropriate O-glycosylation is essential for cancer progression and metastasis [24, 26-28]. Surprisingly, the effects of O-glycosylation on the TrkA receptor, the crucial RTKs for the differentiation of sympathetic and sensory neurons, have not yet been fully investigated in NB. First, we investigated whether 
Table 2. Clinicopathologic and biologic factors affecting survival rate in NB patients.

\begin{tabular}{|c|c|c|c|c|c|c|}
\hline \multirow[t]{2}{*}{ Variable } & \multicolumn{2}{|c|}{ Univariate analysis } & \multicolumn{4}{|c|}{ Multivariate analysis } \\
\hline & $\mathbf{R R}$ & $95 \% \mathrm{Cl}$ & $p$ value & $\mathbf{R R}$ & $95 \% \mathrm{Cl}$ & $p$ value \\
\hline Age at diagnosis: $>1.5$ year versus $\leq 1.5$ year & 4.415 & $2.262-4.415$ & $<0.001^{*}$ & 1.961 & $0.765-5.022$ & 0.161 \\
\hline MYCN: amplified versus non-amplified & 3.867 & 2.413-6.199 & $<0.001^{*}$ & 2.169 & $1.243-3.783$ & $0.006^{*}$ \\
\hline C1GALT1 protein expression: low versus high expression & 3.142 & $1.488-6.634$ & $0.003^{*}$ & 2.329 & $1.056-5.136$ & $0.036^{*}$ \\
\hline Histology: undifferentiated versus differentiated & 3.119 & $1.642-5.923$ & $0.001^{*}$ & 2.071 & $1.027-4.174$ & $0.042^{*}$ \\
\hline
\end{tabular}

${ }^{*} p<0.05$.
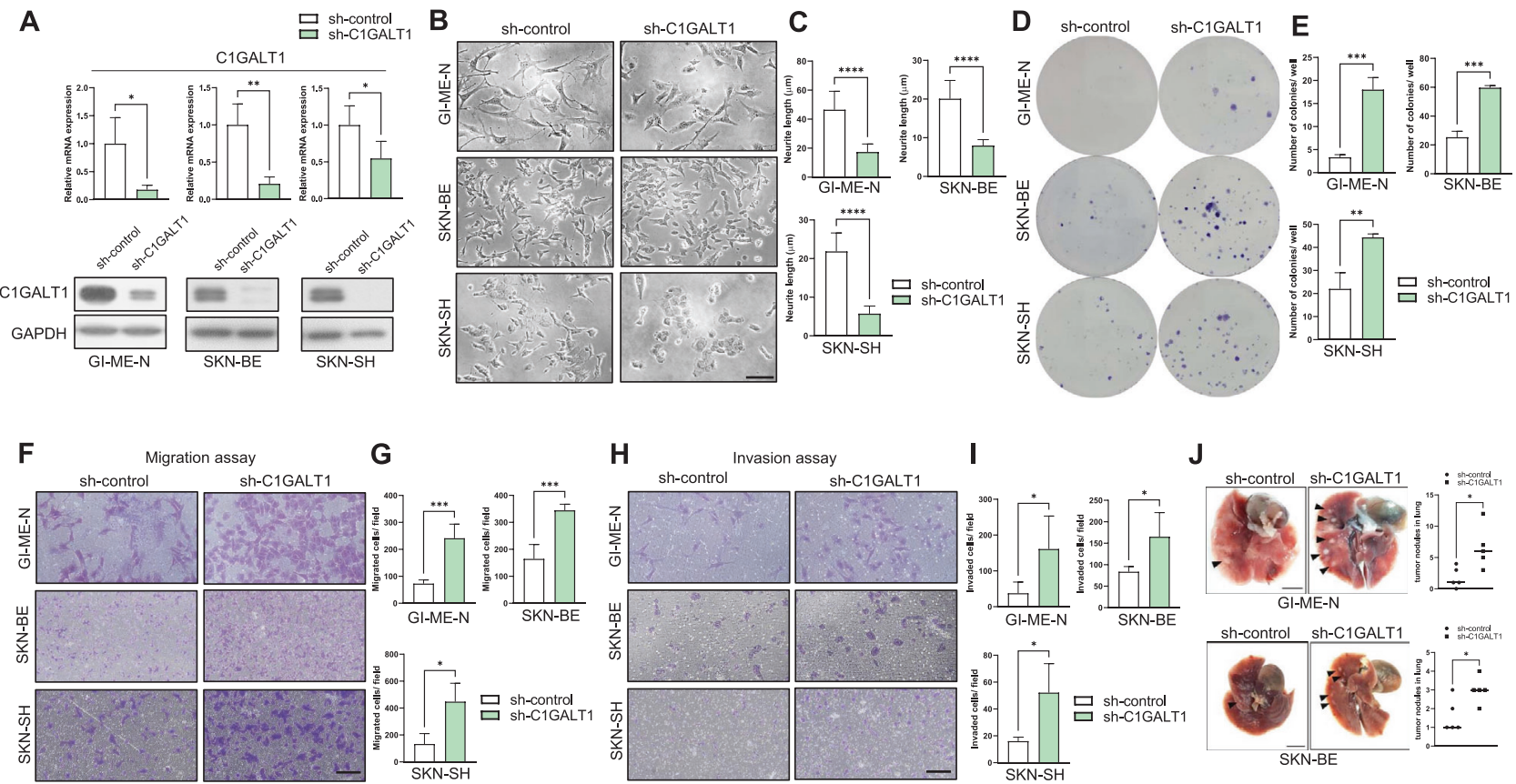

Fig. 2 Downregulation of C1GALT1 promoted the malignant phenotypes of NB cells in vitro and in vivo. A qRT-PCR and Western blot analysis of C1GALT1 expression in GI-ME-N, SK-N-BE, and SK-N-SH cells infected with shC1GALT1 or negative control lentivirus. B Representative images showing neurite outgrowth of NB cells transfected with control shRNA or C1GALT1 shRNA lentivirus for 3 d were collected under a phase-contrast microscope. Scale bar indicates $100 \mu \mathrm{m}$. Arrowheads indicate neurite outgrowth. C Bars represent the mean of three independent experiments. D Representative pictures showed the ability of colony formation in NB cells infected with shC1GALT1 or negative control lentivirus. E Bars represent the mean number of colonies $(n=3)$ (magnification, $\times 100){ }^{* *} p<0.01,{ }^{* * *} p<0.001$. F Representative pictures showed transwell migration assay in NB cells infected with shC1GALT1 or negative control lentivirus. G Bars represent the mean number of migrated cells $(n=3)$. Scale bar indicates $100 \mu \mathrm{m}$. $\mathbf{H}$ Representative pictures showed transwell invasion assay in NB cells infected with shC1GALT1 or negative control lentivirus. I Bars represent the mean number of invaded cells $(n=3)$. Scale bar indicates $100 \mu \mathrm{m}$. J Representative macroscopic images and quantification showed the metastatic lung nodules of mice intracardially injected with NB cells infected with shC1GALT1 or negative control lentivirus. Arrowheads indicate the metastatic nodules. Scale bar indicates 5 mm. ${ }^{*} p<0.05$, ${ }^{* *} p<0.01,{ }^{* * *} p<0.001,{ }^{* * *} p<0.0001$.

manipulating C1GALT1 expression affects O-glycosylation in NB cells. Flow cytometry revealed that knockdown of C1GALT1 enhanced Vicia villosa agglutinin (VVA) lectin binding to cell surfaces in GI-LN-M and SK-N-BE cells (Fig. 3A). To further confirm the presence of C1GALT1-mediated O-glycosylation on TrkA, C1GALT1 knockdown or overexpressing NB cells were analyzed using VVA, GSL-I, and PNA lectin pull-down and TrkA immunoblotting assays, respectively. The results showed that knockdown of C1GALT1 in NB cells increased TrkA pulled down through VVA and GSL-I lectin beads. On the other hand, overexpression of C1GALT1 in NB cells increased TrkA pulled down through PNA lectin beads. The effect of VVA and GSL-I lectin binding is more obvious after enzymatic desialylation. These results indicated that modifying the C1GALT1 expression indeed alters O-glycosylation of TrkA in NB cells. (Fig. 3B, Supplementary Fig. 3). However, the effects of C1GALT1-mediated O-glycosylation on TrkA are unclear. We first analyzed the levels of TrkA mRNA in mock and shC1GALT1 transfectants. The real-time RT-PCR analysis showed no significant change in the mRNA levels of TrkA in shC1GALT1 transfected NB cells (Supplementary Fig. 4). We further performed Western blotting and immunofluorescence staining to detect the expression and examine the localization of TrkA protein in C1GALT1 knockdown cells, respectively. The Western blotting results showed that TrkA protein expression level was decreased in both C1GALT1 knockdown GI-ME-N and SK-N-BE cells (Fig. 3C). The representative fluorescent images taken using confocal microscopy demonstrated that TrkA was predominantly observed on intracellular vesicles in C1GALT1 knockdown transfectants (Fig. 3D). Moreover, flow cytometry consistently provided evidence that silencing C1GALT1 suppressed the localization of TrkA on the 
A

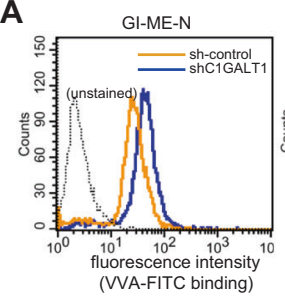

D

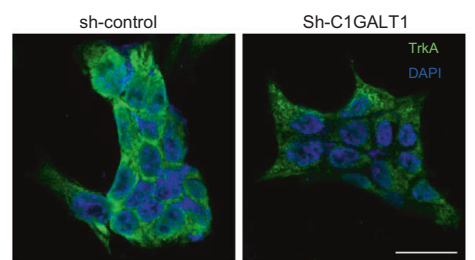

$E$

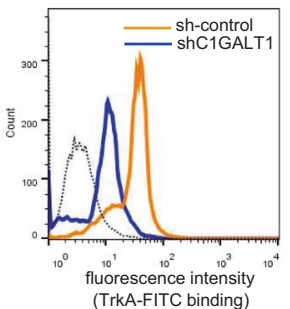

B
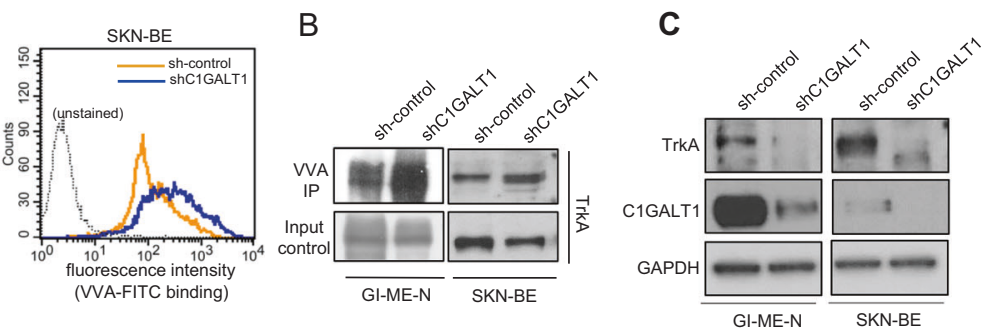

$\mathbf{F}$
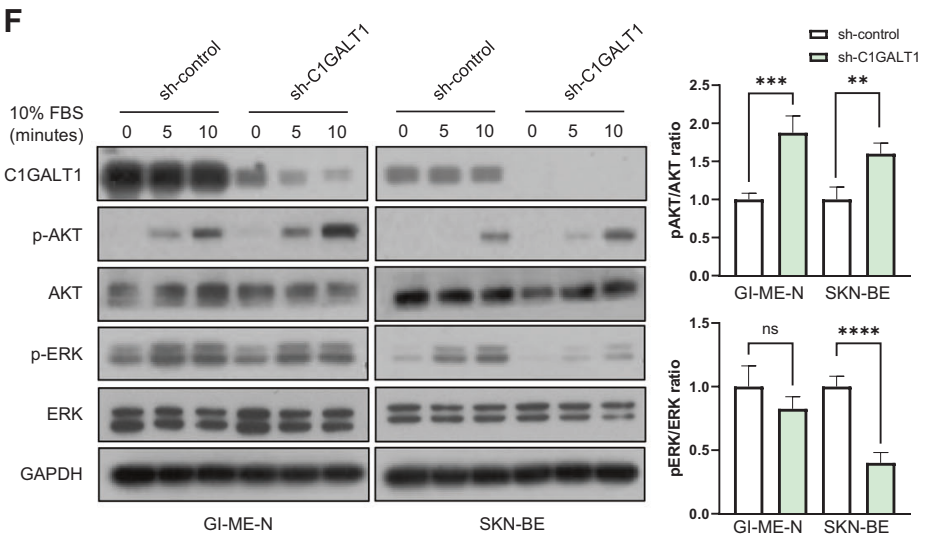

Fig. 3 Downregulation of C1GALT1-mediated O-glycan change regulates the protein expression and localization of TrkA and its downstream signaling. A-B C1GALT1 could modify the Tn antigen expression on TrkA in NB cells. A Representative histogram plots from three independent experiments are shown for flow cytometry with FITC-VVA lectin in GI-ME-N and SK-N-BE cells infected with shC1GALT1 or negative control lentivirus. B Total lysates were pulled down (PD) using VVA agarose beads and then immunoblotted with a TrkA antibody. C Expression of TrkA, C1GALT1, and GAPDH in whole lysates was shown by Western blotting. D-E Inhibition of C1GALT1 could regulate the localization of TrkA. D Representative immunofluorescence images taken from confocal microscope show the localization of TrkA (green) in cells. DAPI (blue) was used to stain the nucleus. Scale bar indicates $50 \mu \mathrm{m}$. E Representative histogram plots showed TrkA expression levels at the cell surface measured by flow cytometry. F C1GALT1 knockdown decreased phosphorylation of ERK, but increased phosphorylation of AKT in GI-ME-N and SK-N-BE cells. Serum-starved cells were reintroduced with 10\% FBS for different times as indicated. Western blots show the expression of AKT, p-AKT, ERK, p-ERK, C1GALT1, and GAPDH $(n=3)$. The bar diagrams of the ratios of p-AKT/total AKT and p-ERK/total ERK were shown in the right panel. ${ }^{* *} p<0.01,{ }^{* * *} p<0.001,{ }^{* * *} p<0.0001$.

surface in SKN-BE cells (Fig. 3E). Because TrkA activates several signaling pathways responsible for the differentiation and proliferation of NB cells, we, therefore, hypothesized that the C1GALT1-mediated alteration in TrkA O-glycans may affect its downstream signaling. The immunoblotting results and the relative quantification of the ratios of $p$-AKT/total AKT and $p$ ERK/total ERK showed that the level of phosphorylated AKT was significantly increased in C1GALT1 knockdown cells, but attenuated the level of phosphorylated ERK, suggesting that inhibition of C1GALT1 is involved in sustained AKT signaling downstream of TrkA (Fig. 3F).

\section{TrkA signaling pathways are involved in the phenotypic changes mediated by C1GALT1}

We next aimed to determine whether upregulation of C1GALT1 may contribute to the antitumor phenotypes of NB cells and promote neurite outgrowth through TrkA pathway. Overexpression of C1GALT1 increased the protein levels of TrkA (Fig. 4A) and promoted the differentiation of NB cells with increased the growth of neurites, whereas knockdown of TrkA inhibited C1GALT1induced neuronal differentiation (Fig. 4B). Moreover, the inhibitory effects of migration and invasion in C1GALT1-overexpressing NB cells were blocked by shRNA-mediated TrkA downregulation (Fig. $4 C, D)$. Since the activation of ERK and AKT signaling are the major response of NGF/TrkA for neuronal cell differentiation and proliferation, respectively [39], we next analyzed the phosphorylation levels of ERK and AKT in the C1GALT1-overexpressing and TrkA knockdown NB cells. Similar as C1GALT1 knockdown, the bar diagram of the ratios of $p$-AKT/total AKT and p-ERK/total ERK indicated that TrkA knockdown also decreased C1GALT1-induced phosphorylation of ERK, but reversely increased phosphorylation of AKT in SK-N-SH cells (Fig. 4E). Collectively, these results indicated that TrkA signaling pathways are at least partially involved in C1GALT1-mediated malignant phenotypes of NB cells.

\section{DISCUSSION}

In this study, the analysis of tumor samples from a separate, larger cohort of NB patients demonstrated that C1GALT1 was highly expressed in ganglion cells and well-differentiated NB; and that C1GALT1 high expression correlated with clinicopathologic features including age, disease stage, histologic classification, and the expression of TrkA. C1GALT1 high expression is an independent prognostic factor for better survival outcomes in NB patients, and could provide complementary prognostic information in addition to age of patients, differentiation status of tumor, MYCN amplification status, and clinical stages. Knockdown of C1GALT1 promoted malignant properties of NB cells in vitro and enhanced the pulmonary metastases of NB in vivo. Moreover, our results demonstrated that $\mathrm{O}$-glycosylation is a critical regulatory factor for TrkA stability and signaling. Silencing of C1GALT1 truncated O-glycans on TrkA, inhibited the presence of TrkA on the plasma membrane, and enhanced migration and invasion in NB cells, whereas overexpression of C1GALT1 promoted cell differentiation and suppressed the migration and invasion of NB cells. Furthermore, we also found that the effect of C1GALT1 knockdown on cell malignant phenotypes was reproduced by silencing TrkA in C1GALT1-overexpressing NB cells. These results 
A

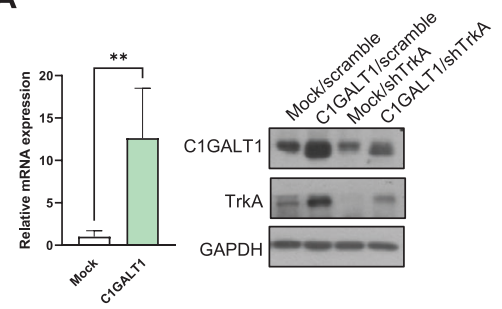

C
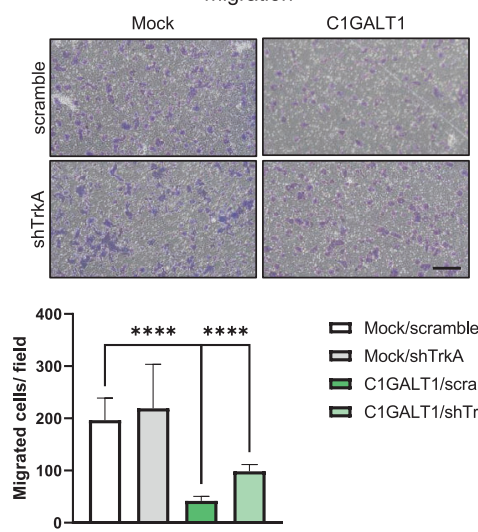

D

D Mock/scramble

ㅁ Mock/shTrkA

口 C1GALT1/scramble

口 C1GALT1/shTrkA
B
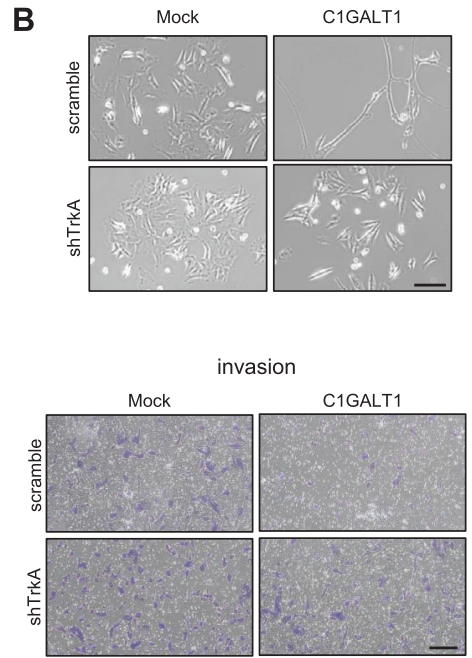

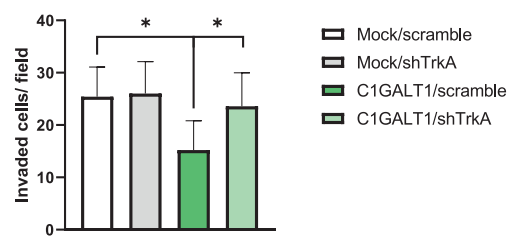

ㅁ Mock/scramble

口 Mock/shTrkA

口 C1GALT1/scramble

口 C1GALT1/shTrkA

Fig. 4 Overexpression of C1GALT1 mediates antitumor effects on NB cells through TrkA. A qRT-PCR analysis showed the mRNA expression levels of C1GALT1 in mock and C1GALT1-overexpressing SK-N-SH cells. Western blot analysis showed the protein expression levels of C1GALT1, TrkA and GAPDH in SK-N-SH transfectants infected with shTrkA or negative control lentivirus. B-D C1GALT1 overexpressionmediated antitumor effects were attenuated by silencing TrkA. B Representative images showed neurite outgrowth of SK-N-SH transfectants infected with shTrkA or negative control lentivirus for $3 \mathrm{~d}$ photographed under a phase-contrast microscope. Scale bar indicates $100 \mu \mathrm{m}$. Arrowheads indicate neurite outgrowth. Bars represent the mean of three independent experiments. C Representative pictures showed the ability of transwell migration in SK-N-SH transfectants infected with shTrkA or negative control lentivirus. Bars (lower panel) represent the mean number of migrated cells $(n=3$, scale bar indicates $100 \mu \mathrm{m})$. D Representative pictures showed Matrigel invasion assay in SK-N-SH transfectants infected with shTrkA or negative control lentivirus. Bars (lower panel) represent the mean number of invaded cells $(n=3$, scale bar indicates $100 \mu \mathrm{m}$ ). E C1GALT1 overexpression-mediated changes in AKT and ERK phosphorylation were reversed by silencing TrkA. Serumstarved cells were reintroduced with $10 \%$ FBS for different times as indicated. Western blots show the expression of AKT, p-AKT, ERK, p-ERK, C1GALT1, and GAPDH $(n=3)$. The bar diagrams of the ratios of $\mathrm{p}-\mathrm{AKT} /$ total AKT and $\mathrm{p}$-ERK/total ERK were shown in the right panel. ${ }^{*} p<0.05$, ${ }^{* *} p<0.01,{ }^{* * *} p<0.001,{ }^{* * * *} p<0.0001$.

suggest that C1GALT1-mediated changes in TrkA O-glycosylation partly regulate the malignant behaviors of NB cells. This is the first study to demonstrate the effect of O-glycosylation in TrkA signaling in NB. Our findings highlight the prognostic role of C1GALT1 in NB, and provide novel information of C1GALT1 and TrkA on the pathogenesis of NB, which could further help in developing new therapeutic targets or strategies for NB in the future.

RTKs mediate intracellular signaling networks that are involved in the progression of tumorigenesis by promoting cell proliferation, differentiation, survival and cell migration [40]. NB is a clinically heterogeneous pediatric cancer of the sympathetic nervous system that originates from neural crest cells. At present, current evidence suggests that the RTK family of neurotrophin receptors play a critical role in NB development [41]. Particularly, NB tumors expressing TrkA are prone to spontaneous regression or differentiation and strongly correlated with low stage in the clinical relevance [41,42]. Interestingly, the results of our study revealed that TrkA expression was positively correlated with C1GALT1 ( $p=0.001$, Supplementary Table 1$)$ in NB tumors, and C1GALT1 high expression was significantly associated with differentiated tumor histology (Table 1). The Kaplan-Meier analysis in our NB cohort showed that patients with high expression of C1GALT1 had a better survival probability compared with those with low expression of C1GALT1. The multivariate Cox regression analysis in our NB cohort also indicated that C1GALT1 high expression was a significant independent predictor of better survival. Therefore, our clinical data strongly suggested that
C1GALT1 could be a potential good prognostic marker for NB patients and the underlying mechanism by which C1GALT1 affects phenotypic changes of NB may be through TrkA signaling.

Several studies have demonstrated that N-glycosylation is of crucial importance in ligand binding, kinase activity, and the determination of the proper conformation and translocation of the RTKs [14, 43-45]. In addition, our previous studies also identified the stimulatory effect of C1GALT1-mediated O-glycosylation on RTK activity such as EPHA2, integrin, and MET in several adult cancers and C1GALT1 high expression was associated with poor survival of patients in these studies [24, 26-28, 46]. Interestingly, high expression of C1GALT1 in tumor tissues predicts a better prognosis in NB in our current study. Another study showed loss of C1GALT1 in pancreatic cancer is correlated with poor prognosis and metastasis [47]. These observations indicate that the association of C1GALT1 expression and survival in cancer patients is highly dependent on tumor types. In some tumors, C1GALT1 plays a tumor-promoting role, while in others C1GALT1 is tumor-suppressive. In addition, the role of C1GALT1mediated O-glycosylation in TrkA has not yet been investigated in the literature and we, therefore, focused on this issue. Our data showed that silencing C1GALT1 increased VVA and GSL-I lectin binding to TrkA, indicating enriched short O-glycans (Tn antigens) on TrkA. C1GALT1 knockdown decreased the protein expression level of TrkA and its presence on the plasma membrane. Our data provided the first experimental evidence for the existence of GalNAc-type O-glycans on TrkA and altered O-glycan structures can regulate TrkA signaling. Supportively, the NetOglyc server 
developed by Henrik Clausen's team predicts several consecutive O-glycosylation sites (366-368) in TrkA [48]. Moreover, the inhibitory effects of C1GALT1 overexpression on cell migration, invasion, and neurite outgrowth were countervailed by TrkA knockdown. These results suggest that silencing C1GALT1 alters O-glycans on TrkA to decrease its protein level and consequently promotes malignant phenotypes in NB cells.

After the binding of neurotrophic factors, activated Trk receptors will participate in various intracellular signaling pathways, such as extracellular signal-regulated kinase (ERK), phosphatidylinositol 3-kinase (PI3K), Protein kinase $B$ (AKT) and phospholipase $C_{\gamma}(P L C-\gamma)$ mediated signaling pathways, to promote cell differentiation, growth, and survival, as well as to change synaptic plasticity and cell motility $[13,14,49]$. Indeed, our Western blotting data showed that C1GALT1 knockdown decreased phospho-ERK levels, but increased phospho-AKT levels in NB cells, whereas overexpression of C1GALT1 elevated phospho-ERK levels, but decreased phospho-AKT levels. Moreover, the effects of C1GALT1 overexpression on the regulation of ERK and AKT pathways were abrogated by silencing TrkA with shRNA. The signaling patterns are consistent with in vitro phenotypes, suggesting that C1GALT1-mediated O-glycosylation can determine TrkA signaling for neuronal differentiation. These findings support the regulatory effect of O-glycans on RTK functions and imply regulating O-glycosylation on TrkA as a potent therapeutic strategy for NB.

Taken together, our study showed that C1GALT1 high expression is an independent prognostic factor and predicts better survival outcomes for NB patients, complementary to age of patients, differentiation status of tumor, MYCN status, and clinical stages. Silencing C1GALT1 decreases TrkA expression and promotes malignant phenotypes in NB in vitro and in vivo. Particularly, the in vitro finding that overexpression of C1GALT1 increases the neurite outgrowth of NB cells is consistent with the high expression of C1GALT1 observed in the NB patients with differentiated tumor histology. These results derived from the clinical analysis of NB patients to phenotypic cellular assays provide new insights that C1GALT1 is considered to exert antitumor effects in NB. Our data provided evidence for the existence of GalNAc-type O-glycans on TrkA and altered O-glycan structures by C1GALT1 can regulate TrkA signaling in NB. This is the first study to demonstrate the effect of O-glycosylation in TrkA signaling in NB. Results of this study shed light on the novel prognostic role of C1GALT1 in NB, in contrast with adult cancers, and provide new information of C1GALT1 and TrkA on the pathogenesis of NB, which could further help to develop new therapeutic strategies for NB in the future.

\section{DATA AVAILABILITY}

The datasets used and/or analyzed during the current study are available from the corresponding author on reasonable request.

\section{REFERENCES}

1. Gurney JG, Severson RK, Davis S, Robison LL. Incidence of cancer in children in the United States. Sex-, race-, and 1-year age-specific rates by histologic type. Cancer. 1995;75:2186-95.

2. Grovas A, Fremgen A, Rauck A, Ruymann FB, Hutchinson CL, Winchester DP, et al. The National Cancer Data Base report on patterns of childhood cancers in the United States. Cancer. 1997;80:2321-32.

3. Maris JM, Hogarty MD, Bagatell R, Cohn SL. Neuroblastoma. Lancet. 2007;369:2106-20.

4. Pritchard J, Hickman JA. Why does stage $4 \mathrm{~s}$ neuroblastoma regress spontaneously? Lancet. 1994;344:869-70.

5. Smith MA, Seibel NL, Altekruse SF, Ries LA, Melbert DL, O'Leary M, et al. Outcomes for children and adolescents with cancer: challenges for the twenty-first century. J Clin Oncol. 2010;28:2625-34.
6. Brodeur GM. Neuroblastoma: biological insights into a clinical enigma. Nat Rev. 2003;3:203-16.

7. Pinto NR, Applebaum MA, Volchenboum SL, Matthay KK, London WB, Ambros PF, et al. Advances in risk classification and treatment strategies for neuroblastoma. J Clin Oncol. 2015;33:3008-17.

8. Brodeur GM, Seeger RC, Schwab M, Varmus HE, Bishop JM. Amplification of $\mathrm{N}$-myc in untreated human neuroblastomas correlates with advanced disease stage. Science. 1984;224:1121-4.

9. Seeger RC, Brodeur GM, Sather H, Dalton A, Siegel SE, Wong KY, et al. Association of multiple copies of the $\mathrm{N}$-myc oncogene with rapid progression of neuroblastomas. N Engl J Med. 1985;313:1111-6.

10. Higashi M, Sakai K, Fumino S, Aoi S, Furukawa T, Tajiri T. The roles played by the MYCN, Trk, and ALK genes in neuroblastoma and neural development. Surg Today. 2019;49:721-7.

11. Snider WD. Functions of the neurotrophins during nervous system development: what the knockouts are teaching us. Cell. 1994;77:627-38.

12. Cocco E, Scaltriti M, Drilon A. NTRK fusion-positive cancers and TRK inhibitor therapy. Nat Rev Clin Oncol. 2018;15:731-47.

13. Huang EJ, Reichardt LF. Trk receptors: roles in neuronal signal transduction. Annu Rev Biochem. 2003;72:609-42.

14. Watson FL, Porcionatto MA, Bhattacharyya A, Stiles CD, Segal RA. TrkA glycosylation regulates receptor localization and activity. J Neurobiol. 1999;39:323-36.

15. Pinho SS, Reis CA. Glycosylation in cancer: mechanisms and clinical implications. Nat Rev. 2015;15:540-55.

16. Jensen $\mathrm{PH}$, Kolarich $\mathrm{D}$, Packer NH. Mucin-type O-glycosylation-putting the pieces together. FEBS J. 2010;277:81-94.

17. Ten Hagen KG, Fritz TA, Tabak LA. All in the family: the UDP-GalNAc:polypeptide $\mathrm{N}$-acetylgalactosaminyltransferases. Glycobiology. 2003;13:1R-16R.

18. Tran DT, Ten Hagen KG. Mucin-type O-glycosylation during development. J Biol Chem. 2013;288:6921-9.

19. Kudo T, Sato T, Hagiwara K, Kozuma Y, Yamaguchi T, Ikehara Y, et al. C1galt1deficient mice exhibit thrombocytopenia due to abnormal terminal differentiation of megakaryocytes. Blood. 2013;122:1649-57.

20. Fu J, Wei B, Wen T, Johansson ME, Liu X, Bradford E, et al. Loss of intestinal core 1-derived O-glycans causes spontaneous colitis in mice. J Clin Invest. 2011;121:1657-66.

21. Alexander WS, Viney EM, Zhang JG, Metcalf D, Kauppi $M$, Hyland $C D$, et al. Thrombocytopenia and kidney disease in mice with a mutation in the C1galt1 gene. Proc Natl Acad Sci USA. 2006;103:16442-7.

22. Fu C, Zhao H, Wang Y, Cai H, Xiao Y, Zeng Y, et al. Tumor-associated antigens: Tn antigen, sTn antigen, and T antigen. HLA. 2016;88:275-86.

23. Chou $\mathrm{CH}$, Huang MJ, Chen $\mathrm{CH}$, Shyu MK, Huang J, Hung JS, et al. Up-regulation of C1GALT1 promotes breast cancer cell growth through MUC1-C signaling pathway. Oncotarget. 2015;6:6123-35.

24. Wu YM, Liu CH, Huang MJ, Lai HS, Lee PH, Hu RH, et al. C1GALT1 enhances proliferation of hepatocellular carcinoma cells via modulating MET glycosylation and dimerization. Cancer Res. 2013;73:5580-90.

25. Liu $\mathrm{CH}$, Hu RH, Huang MJ, Lai IR, Chen CH, Lai HS, et al. C1GALT1 promotes invasive phenotypes of hepatocellular carcinoma cells by modulating integrin beta1 glycosylation and activity. PLoS ONE. 2014;9:e94995.

26. Hung JS, Huang J, Lin YC, Huang MJ, Lee PH, Lai HS, et al. C1GALT1 overexpression promotes the invasive behavior of colon cancer cells through modifying O-glycosylation of FGFR2. Oncotarget. 2014;5:2096-106.

27. Lee PC, Chen ST, Kuo TC, Lin TC, Lin MC, Huang J, et al. C1GALT1 is associated with poor survival and promotes soluble Ephrin A1-mediated cell migration through activation of EPHA2 in gastric cancer. Oncogene. 2020;39:2724-40.

28. Lin MC, Chien PH, Wu HY, Chen ST, Juan HF, Lou PJ, et al. C1GALT1 predicts poor prognosis and is a potential therapeutic target in head and neck cancer. Oncogene. 2018;37:5780-93.

29. Kattner P, Strobel H, Khoshnevis N, Grunert M, Bartholomae S, Pruss M, et al. Compare and contrast: pediatric cancer versus adult malignancies. Cancer Metastasis Rev. 2019;38:673-82.

30. Shimada H. The International Neuroblastoma Pathology Classification. Pathologica. 2003;95:240-1.

31. Peuchmaur M, d'Amore ES, Joshi VV, Hata J, Roald B, Dehner LP, et al. Revision of the International Neuroblastoma Pathology Classification: confirmation of favorable and unfavorable prognostic subsets in ganglioneuroblastoma, nodular. Cancer. 2003;98:2274-81.

32. Brodeur GM, Pritchard J, Berthold F, Carlsen NL, Castel V, Castelberry RP, et al. Revisions of the international criteria for neuroblastoma diagnosis, staging, and response to treatment. J Clin Oncol. 1993;11:1466-77.

33. Tsai HY, Hsi BL, Hung IJ, Yang CP, Lin JN, Chen JC, et al. Correlation of MYCN amplification with MCM7 protein expression in neuroblastomas: a chromogenic in situ hybridization study in paraffin sections. Hum Pathol. 2004;35:1397-403. 
34. Chang $\mathrm{HH}$, Chen $\mathrm{CH}$, Chou $\mathrm{CH}$, Liao YF, Huang MJ, Chen $\mathrm{YH}$, et al. beta-1,4Galactosyltransferase III enhances invasive phenotypes via beta1-integrin and predicts poor prognosis in neuroblastoma. Clin Cancer Res. 2013;19:1705-16.

35. Chang HH, Liu YL, Lu MY, Jou ST, Yang YL, Lin DT, et al. A multidisciplinary team care approach improves outcomes in high-risk pediatric neuroblastoma patients. Oncotarget. 2017:8:4360-72.

36. Chang HH, Tseng YF, Lu MY, Yang YL, Chou SW, Lin DT, et al. MYCN RNA levels determined by quantitative in situ hybridization is better than MYCN gene dosages in predicting the prognosis of neuroblastoma patients. Mod Pathol. 2020;33:531-40.

37. Chang HH, Lu MY, Yang YL, Chou SW, Lin DT, Lin KH, et al. The prognostic roles of and correlation between ALK and MYCN protein expression in neuroblastoma. J Clin Pathol. 2020;73:154-61.

38. Nakagawara A, Arima M, Azar CG, Scavarda NJ, Brodeur GM. Inverse relationship between trk expression and $\mathrm{N}$-myc amplification in human neuroblastomas. Cancer Res. 1992;52:1364-8.

39. Khotskaya YB, Holla VR, Farago AF, Mills Shaw KR, Meric-Bernstam F, Hong DS. Targeting TRK family proteins in cancer. Pharm Ther. 2017;173:58-66.

40. Lemmon MA, Schlessinger J. Cell signaling by receptor tyrosine kinases. Cell 2010;141:1117-34.

41. Brodeur GM, Minturn JE, Ho R, Simpson AM, lyer R, Varela CR, et al. Trk receptor expression and inhibition in neuroblastomas. Clin Cancer Res. 2009;15:3244-50.

42. Combaret V, Gross N, Lasset C, Balmas K, Bouvier R, Frappaz D, et al. Clinical relevance of TRKA expression on neuroblastoma: comparison with N-MYC amplification and CD44 expression. Br J Cancer. 1997;75:1151-5.

43. Lopez Sambrooks C, Baro M, Quijano A, Narayan A, Cui W, Greninger P, et al. Oligosaccharyltransferase inhibition overcomes therapeutic resistance to EGFR tyrosine kinase inhibitors. Cancer Res. 2018;78:5094-106.

44. Kaszuba K, Grzybek M, Orlowski A, Danne R, Rog T, Simons K, et al. $\mathrm{N}$-Glycosylation as determinant of epidermal growth factor receptor conformation in membranes. Proc Natl Acad Sci USA. 2015;112:4334-9.

45. Perez AG, Andrade-Da-Costa J, De Souza WF, De Souza Ferreira M, Boroni M, De Oliveira IM, et al. Nglycosylation and receptor tyrosine kinase signaling affect claudin3 levels in colorectal cancer cells. Oncol Rep. 2020;44:1649-61.

46. Kuo TC, Wu MH, Yang SH, Chen ST, Hsu TW, Jhuang JY, et al. C1GALT1 high expression is associated with poor survival of patients with pancreatic ductal adenocarcinoma and promotes cell invasiveness through integrin alphav. Oncogene. 2021;40:1242-54.

47. Chugh S, Barkeer S, Rachagani S, Nimmakayala RK, Perumal N, Pothuraju R, et al. Disruption of C1galt1 gene promotes development and metastasis of pancreatic adenocarcinomas in mice. Gastroenterology. 2018;155:1608-24.

48. Steentoft C, Vakhrushev SY, Joshi HJ, Kong Y, Vester-Christensen MB, Schjoldager $\mathrm{KT}$, et al. Precision mapping of the human O-GalNAc glycoproteome through SimpleCell technology. EMBO J. 2013;32:1478-88.

49. Uren RT, Turnley AM. Regulation of neurotrophin receptor (Trk) signaling: suppressor of cytokine signaling 2 (SOCS2) is a new player. Front Mol Neurosci. 2014;7:39.

\section{ACKNOWLEDGEMENTS}

The authors wish to thank Dr. Rong-Long Chen from the Koo Foundation Sun Yat-Sen Cancer Center (Taipei, Taiwan) and the Childhood Cancer Foundation (Taiwan) for designing and maintaining the TPOG neuroblastoma treatment protocols. We are grateful to all the patients and their parents who participated in this study. We would like to acknowledge the service provided by the Flow Cytometric Analyzing and Sorting Core of the First Core Laboratory, National Taiwan University College of Medicine. This study was supported by the Excellent Translational Medicine Research Projects of National Taiwan University College of Medicine, and National Taiwan University Hospital: 107C101-B3, 108C101-83, 109C101-93, NSCCMOH-94-8 and NSCCMOH-131-92 (N.-Y.L.), 111-S0028 (H.-H.C.), and the technical assistance (e.g. the Cell Imaging Core) of the First Core Labs in the National Taiwan University College of
Medicine and Ministry of Science and Technology, R.O.C. 106-2320-B-002-004-MY3 (N.Y.L.), 109-2320-B-002-039 (N.-Y.L.), 108-2314-B-002-154 (H.-H.C.), 109-2314-B-002-129 (H.-H.C.), 110-2314-B-002-088-MY3 (H.-H.C.), and 108-2320-B-002 -064 -MY3 (М.-С.H).

\section{AUTHOR CONTRIBUTIONS}

N.-Y.L.: Methodology, Data curation, Writing - original draft, Funding acquisition. S.-T. C.: Methodology, Data curation. H.-L.C.: Methodology, Data curation. M.-Y.L. Resources, Validation. Y.-L.Y.: Resources, Validation. S.-W.C.: Resources, Validation. D.-T.L.: Resources, Validation. K.-H. L.: Resources, Validation. S.-T.J.: Resources, Validation. W.-M.H.: Resources, Validation, Supervision, Conceptualization. M.-C.H.: Resources, Validation, Supervision, Conceptualization, Writing - review \& editing, Funding acquisition. H.-H.C.: Conceptualization, Methodology, Data curation, Writing -original draft, Writing-review \& editing, Project administration, Funding acquisition.

\section{COMPETING INTERESTS}

The authors declare no competing interests.

\section{ETHICS APPROVAL AND CONSENT TO PARTICIPATE}

Regarding the use of human neuroblastoma tumor samples, written informed consent was obtained from all patients and this study was approved by the Institutional Review Board of the National Taiwan University Hospital (IRB No: 201812005RIND). All mouse experiments were carried out in accordance with a study protocol approved by the National Taiwan University College of Medicine and College of Public Health Institutional Animal Care and Use Committee (IACUC) (No. 20180305).

\section{ADDITIONAL INFORMATION}

Supplementary information The online version contains supplementary material available at https://doi.org/10.1038/s41389-022-00383-w.

Correspondence and requests for materials should be addressed to Hsiu-Hao Chang.

Reprints and permission information is available at http://www.nature.com/ reprints

Publisher's note Springer Nature remains neutral with regard to jurisdictional claims in published maps and institutional affiliations.

\begin{abstract}
(c) (i)
Open Access This article is licensed under a Creative Commons Attribution 4.0 International License, which permits use, sharing, adaptation, distribution and reproduction in any medium or format, as long as you give appropriate credit to the original author(s) and the source, provide a link to the Creative Commons license, and indicate if changes were made. The images or other third party material in this article are included in the article's Creative Commons license, unless indicated otherwise in a credit line to the material. If material is not included in the article's Creative Commons license and your intended use is not permitted by statutory regulation or exceeds the permitted use, you will need to obtain permission directly from the copyright holder. To view a copy of this license, visit http://creativecommons. org/licenses/by/4.0/.
\end{abstract}

(c) The Author(s) 2022 